\title{
DENSITIES IN ARITHMETIC PROGRESSIONS
}

\section{MORRIS GOLDFELD}

Let $S=\left\{s_{1}, s_{2}, \cdots\right\}$ be a set of positive integers. Then the density of $S$ (denoted by $d(S)$ ) is the $\lim _{n \rightarrow \infty} S(n) / n$, if the limit exists, where $S(n)$ is the number of integers in $S$ that are less than or equal to $n$. Clearly, if $A$ is an arithmetic progression of difference $a$, then $d(A)=1 / a$.

If we consider the algebra consisting of all finite unions of arithmetic progressions, then it can easily be shown that the density function is a finitely additive measure on this algebra. The chief obstruction to our knowledge about the density function lies in the fact that the density does not extend to the $\sigma$-algebra. In certain cases, however, it does. This paper is concerned with those cases; and in particular with the arithmetic progressions $A_{i}$ with differences $a_{i}$ satisfying the following condition

$$
d\left(\bar{A}_{i}\right)=\prod\left(1-1 / a_{i}\right)
$$

where the intersection and product run through $i=1,2,3, \cdots$ and where $\bar{A}_{i}$ denotes the complement of $A_{i}$. It will be shown if the preceding condition is satisfied that one can give fairly simple expressions for the density of $\cap \bar{A}_{i}$ in an arithmetic progression, if the density exists in that progression. It seems to be true that if (1) holds then $d\left(\left(\cap \bar{A}_{i}\right) \cap B\right)$ exists for any arithmetic progression $B$ although I do not see how to prove it.

Let $\left\{a_{1}, a_{2}, \cdots\right\}$ be a set of pairwise relatively prime positive integers. Let $A_{i}$ be the set of all positive multiples of $a_{i}$. Put $S=\cap \bar{A}_{i}$. We shall prove the following general theorem on the density of $S \cap B$, where $B$ is the set of all positive multiples of $b$ for an arbitrary positive integer $b$.

THEOREM. Let $b=b^{\prime} q_{1} q_{2} \cdots q_{\text {s }}$ where $b^{\prime}$ is (for each $i$ ) relatively prime to $\left(a_{i} / q_{i}\right)$ and $q_{k}$ is the greatest common divisor of $b$ and $a_{k}$. Then "if

$$
d(S)=\prod\left(1-1 / a_{i}\right)
$$

we must also have

$$
d(B \cap S)=\frac{1}{b^{\prime}} \prod_{k=1}^{8}\left(\frac{a_{k} / q_{k}-1}{a_{k}-1}\right) \prod\left(1-1 / a_{i}\right)
$$

if the density of $B \cap S$ exists.

Received by the editors April 25, 1967. 
Proof. Let $|A|$ denote the number of elements in any set $A$. Also let $I_{n}=\{1,2, \cdots n\}$ be the set containing the first $n$ integers.

Put $S_{r}=\bigcap_{i=1}^{r} \bar{A}_{i}$ and $S_{r}(n)=\left|S_{r} \cap I_{n}\right|$. We consequently have by virtue of the definition of density

$$
d(S)=\lim _{n \rightarrow \infty} \frac{1}{n}\left(\lim _{r \rightarrow \infty} S_{r}(n)\right)
$$

Since $d(S)$ exists (by assumption) it does not matter through what sequence of $n$ we reach our limit, as long as we choose $n$ constantly increasing.

Let $n_{j}=\prod_{i=1}^{j} a_{i}$. We then have,

$$
d(S)=\lim _{j \rightarrow \infty} \frac{1}{b n_{j}}\left(\lim _{r \rightarrow \infty} S_{r}\left(b n_{j}\right)\right) .
$$

But $\bar{A}_{v} \cap I_{b n_{j}}=I_{b n_{j}}$ for $v \geqq b n_{j}$ since $\bar{A}_{i}$ contains all the integers less than $a_{i}$ for each $i$. Hence,

$$
\begin{aligned}
\lim _{r \rightarrow \infty} S_{r}\left(b n_{j}\right) & =S_{b n_{j}}\left(b n_{j}\right) \\
& =S_{j}\left(b n_{j}\right)-X(b, j),
\end{aligned}
$$

where $X(b, j)$ is the error.

On making use of the exclusion-inclusion principle, we see that

$$
S_{j}\left(b n_{j}\right)=b \prod_{i=1}^{j}\left(a_{i}-1\right) .
$$

Putting all this back into the expression for density yields

$$
d(S)=\prod\left(1-1 / a_{i}\right)-\lim _{j \rightarrow \infty} X(b, j) / n_{j},
$$

and since $d(S)=\prod\left(1-1 / a_{i}\right)$ by assumption, this makes

$$
\lim _{j \rightarrow \infty} X(b, j) / n_{j}=0 .
$$

Now, let $S_{r}(B, n)=\left|S_{r} \cap B \cap I_{n}\right|$. Then

$$
\begin{aligned}
d(B \cap S) & =\lim _{j \rightarrow \infty} \frac{1}{b n_{j}}\left(\lim _{r \rightarrow \infty} S_{r}\left(B, b n_{j}\right)\right) \\
& =\lim _{j \rightarrow \infty} \frac{1}{b n_{j}}\left(S_{b n_{j}}\left(B, b n_{j}\right)\right)
\end{aligned}
$$

as before. We now put 


$$
S_{b n_{j}}\left(B, b n_{j}\right)=S_{j}\left(B, b n_{j}\right)-X^{1}(b, j)
$$

where $X^{1}(b, j) \leqq X(b, j)$ for all $j$. Making use of the exclusion-inclusion principle once again, we see that

$$
S_{j}\left(B, b n_{j}\right)=\frac{b}{b^{\prime}} \prod_{k=1}^{s} \frac{a_{k} / q_{k}-1}{a_{k}-1} \prod_{i=1}^{j}\left(a_{i}-1\right) .
$$

Therefore,

$$
d(B \cap S)=\frac{1}{b^{\prime}} \prod_{k=1}^{s} \frac{a_{k} / q_{k}-1}{a_{k}-1} \prod\left(1-1 / a_{i}\right)-\lim _{j \rightarrow \infty} X(b, j) / n_{j},
$$

and since $X^{1}(b, j) \leqq X(b, j)$, this makes (by equation (2))

$$
\lim _{j \rightarrow \infty} X^{1}(b, j) / n_{j}=0 .
$$

Corollary (1). Let $\left\{a_{1}, a_{2}, \cdots\right\}$ be a set of pairwise relatively positive integers with $A_{i}$ denoting the set of positive multiples of $a_{i}$. Also, let $X_{k}$ denote the number of integers less than $\prod_{1}^{k} a_{i}$ which are not divisible by any $a_{i}, 0<i \leqq k$, and divisible by some $a_{j}$ where $k<j \leqq \prod_{1}^{k} a_{i}$. Then if (1) holds, we must also have

$$
\lim _{k \rightarrow \infty} \frac{X_{k}}{\prod_{1}^{k} a_{i}}=0 .
$$

Proof. The proof follows immediately from the proof of the preceding theorem (by equation (2)) if we can show that

$$
X_{k}=S_{k}\left(n_{k}\right)-S_{n_{k}}\left(n_{k}\right)=X(1, k) .
$$

But the right side of (3) is just what we mean by $X_{k}$.

Q.E.D.

As an application of the theorem we consider the problem of finding the density of squarefree integers in an arithmetic progression.

Corollary (2). Let $B=\{b, 2 b, 3 b, \cdots\}$ where $b$ is squarefree and can be factored into primes $q_{1} q_{2} \cdots q_{s}$; then we have (for $S=$ squarefree integers)

$$
d(B \cap S)=\frac{6}{\pi^{2}} \prod_{i=1}^{s}\left(1 /\left(q_{i}+1\right)\right)
$$

if the density exists.

Proof. Let $A_{i}$ consist of the positive multiples of $q_{i}^{2}$ for $i=1, \cdots, s$. Let $\left\{p_{s+1}, p_{s+2}, \cdots\right\}$ be the set of all primes relatively prime to $b$. 
We let $A_{j}$ denote the set of all positive multiples of $p_{j}^{2}$ for $j>s$. Then $S=\cap \bar{A}_{j}$ is nothing more than the set of squarefree integers. But we already know that $[1$, p. 269]

$$
d(S)=\prod\left(1-1 / p^{2}\right)=6 / \pi^{2},
$$

where the product is over all primes $p$. Hence $S$ satisfies the condition (1). By the result of our theorem, we must therefore have

$$
\begin{aligned}
& d(B \cap S)= \prod_{k=1}^{s} \frac{q_{k}^{2} / q_{k}-1}{q_{k}^{2}-1} \prod\left(1-1 / p^{2}\right) \\
&= \frac{6}{\pi^{2}} \prod 1 /\left(q_{k}+1\right) . \\
& \text { REFERENCE }
\end{aligned}
$$

1. G. H. Hardy and E. M. Wright, Introduction to the theory of numbers, 4th ed., Oxford Univ. Press, London, 1960.

Columbia UNiversity 